\title{
Super-beacons: Open-source probes with spontaneous tuneable blinking compatible with live-cell super-resolution microscopy
}

\author{
Pedro M. Pereira ${ }^{1,2,4}$ \\ Ricardo Henriques ${ }^{1,2}$ \\ Nils Gustafsson ${ }^{1}$ \\ Mark Marsh ${ }^{1}$ \\ Musa M. Mhlanga ${ }^{3}$
}

${ }^{1}$ MRC-Laboratory for Molecular Cell Biology, University College London, London, UK

${ }^{2}$ The Francis Crick Institute, London, UK

${ }^{3}$ Institute of Infectious Disease and Molecular Medicine, Faculty of Health Sciences, University of Cape Town, Cape Town, South Africa

${ }^{4}$ Bacterial Cell Biology, MOSTMICRO, ITQBNOVA, Oeiras, Portugal

\section{Correspondence \\ Ricardo Henriques, MRC-Laboratory for Molecular Cell Biology, University College London, London, UK. \\ Email: r.henriques@ucl.ac.uk}

\section{Present address}

Nils Gustafsson, Department für Physik and CeNS, Ludwig-Maximilians-Universität,

Munich, Germany

\section{Funding information}

Biotechnology and Biological Sciences Research Council, Grant/Award Numbers: BB/ M022374/1, BB/P027431/1, BB/R000697/1; Engineering and Physical Sciences Research Council, Grant/Award Number: EP/ L504889/1; Medical Research Council, Grant/ Award Number: MR/K015826/1; Wellcome Trust, Grant/Award Number: 203276/Z/16/Z

\section{Peer Review}

The peer review history for this article is available at https://publons.com/publon/10.

1111/tra.12728/.

\begin{abstract}
Localization-based super-resolution microscopy relies on the detection of individual molecules cycling between fluorescent and non-fluorescent states. These transitions are commonly regulated by high-intensity illumination, imposing constrains to imaging hardware and producing sample photodamage. Here, we propose single-molecule self-quenching as a mechanism to generate spontaneous photoswitching. To demonstrate this principle, we developed a new class of DNA-based open-source superresolution probes named super-beacons, with photoswitching kinetics that can be tuned structurally, thermally and chemically. The potential of these probes for livecell compatible super-resolution microscopy without high-illumination or toxic imaging buffers is revealed by imaging interferon inducible transmembrane proteins (IFITMs) at sub-100 $\mathrm{nm}$ resolutions.
\end{abstract}

\section{KEYWORDS}

DNA, fluorescence microscopy, fluorescence quenching, molecular-beacons, live-cell, superresolution

\section{SYNOPSIS}

We developed a new class of DNA-based self-quenching open-source super-resolution probes named super-beacons, with photoswitching kinetics that can be tuned structurally, thermally and chemically. These probes

Pedro M. Pereira and Nils Gustafsson equally contributed to this study. alleviate the need for high-intensity illumination and toxic buffers usually needed in single-molecule localization super-resolution approaches. We showcase their applicability in fixed- and live-cell imaging with sub$100 \mathrm{~nm}$ resolutions to explore the organisation of interferon inducible transmembrane proteins (IFTM) at the cell surface. Ab link; Link to antibody of interest. mW.cm-2; Live-cell compatible illumination.

This is an open access article under the terms of the Creative Commons Attribution License, which permits use, distribution and reproduction in any medium,

(c) 2020 The Authors. Traffic published by John Wiley \& Sons Ltd. 


\section{1 | INTRODUCTION}

Super-resolution microscopy (SRM) encapsulates optical imaging methods capable of bypassing the $\sim 250 \mathrm{~nm}$ resolution limit imposed by diffraction. ${ }^{1}$ Their resolving power approaches that of electron microscopy ${ }^{2}$ while keeping the benefits of fluorescence imaging, such as molecular-specific labelling and potential for live-cell imaging. ${ }^{3}$ Single-molecule localization microscopy (SMLM) is a well-establish set of SRM approaches, particularly popular because of their capacity to achieve near-molecular scale resolution $(<50 \mathrm{~nm})$ in relatively simple imaging equipment. ${ }^{4,5}$ SMLM achieves this nanoscale resolution by exploiting fluorophore photoswitching ${ }^{6-9}$ or, more recently, through emission fluctuation in a new family of techniques derived from SMLM. ${ }^{10-12}$ An SMLM acquisition aims to capture individualised fluorophores transitioning between non-emitting and emitting states. The analysis of a sequence of images containing this information then allows the creation of a super-resolution image where the presence and location of these fluorophores can be better-discriminated. ${ }^{13}$ To achieve a considerable resolution increase (10-fold increase when compared to diffraction-limited approaches), these methods rely on specialised labels whose switching kinetics are frequently modulated by intense illumination. ${ }^{14}$ However, this requirement constrains these approaches to microscopes capable of high-intensity illumination and limits compatibility with live-cell imaging due to phototoxicity. ${ }^{3,15,16}$ Exceptions exist, such as genetically encoded fluorophores that photoswitch at low-intensity illumination, these include Dreiklang, ${ }^{17}$ Skylan ${ }^{18}$ and SPOON. ${ }^{19}$ SMLM based on Point Accumulation for
Imaging in Nanoscale Topography (PAINT) exploits an alternative mode of blinking, accomplished through transient binding of fluorescent probes to target molecules. ${ }^{20-22}$ PAINT has the benefit of not requiring high-intensity illumination, as blinking arises from binding kinetics. However, it is often limited to TIRF or Spinning-Disk microscopy, due to the presence of considerable background fluorescence from unbound freely diffusing probes. ${ }^{23}$ Recent studies have started to address this issue. ${ }^{24}$

We propose a new class of super-resolution fluorescent probes for SMLM, dubbed super-beacons (Figure 1). With these molecules, the transition between emitting and non-emitting states is stochastic and independent of illumination. This feature eliminates the need for photodamaging illumination or photoswitching inducing buffers (Figure 1A). At the molecular level this is achieved through a DNA scaffold resembling molecular-beacons (Figure 1B), ${ }^{25}$ altered to achieve high-efficient photoswitching instead of oligonucleotide sensing (Figure 1C). We characterised the photoswitching properties of super-beacons (Figure 1D-E) and demonstrate their applicability for SMLM in live-cell compatible imaging conditions.

\section{2 | RESULTS}

\subsection{Super-beacons probe design}

We hypothesised that fluorophore photoswitching can be induced by promoting transient interactions between a fluorophore-quencher

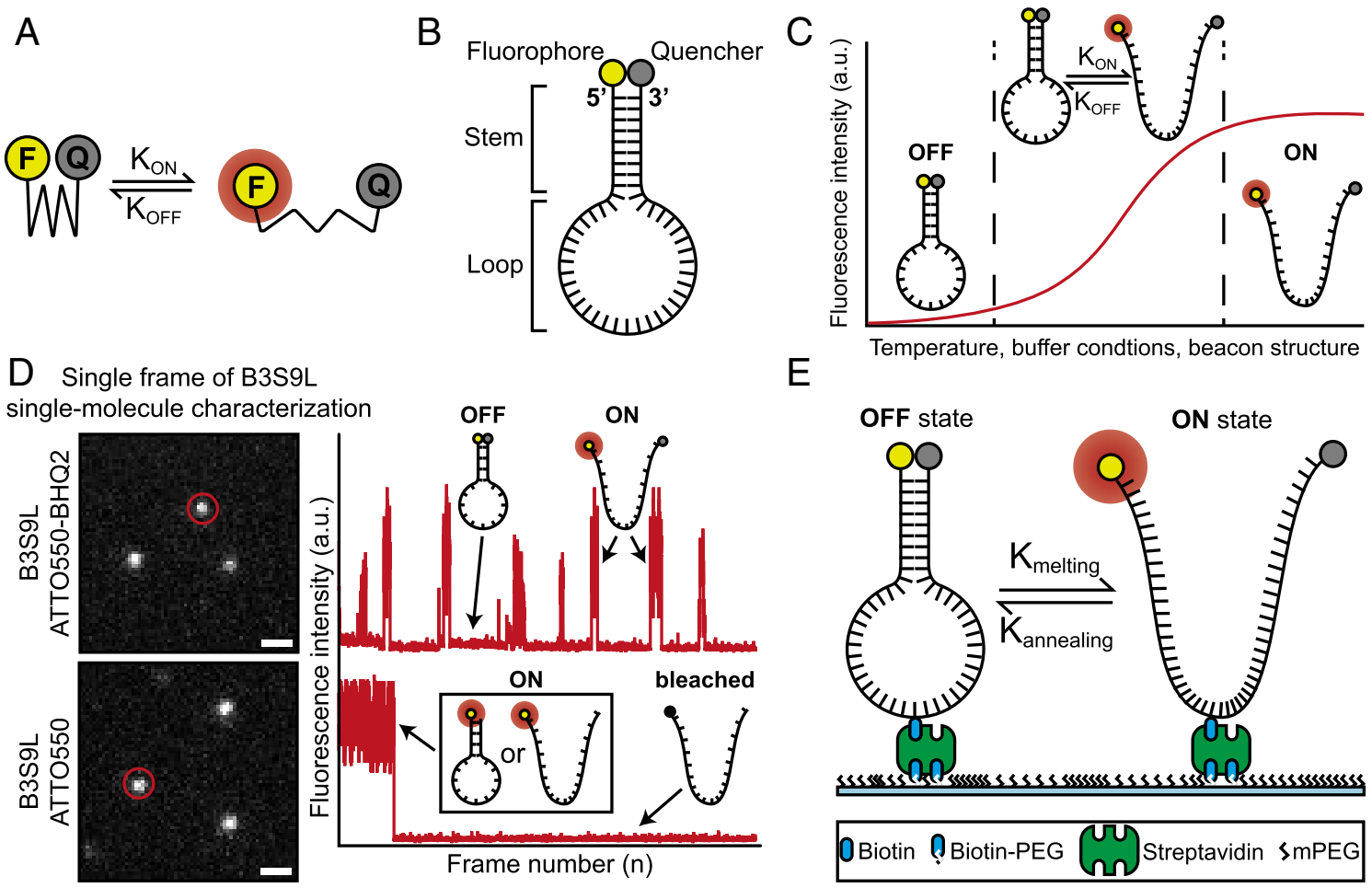

FIGURE 1 Mechanism of super-beacon photoswitching. A, Model of transient quenching-mediated photoswitching. B, Super-beacon structure. C, Intensity plot based on the annealing profile of super-beacons in different conditions. D, (left) Single-molecule in vitro characterisation (Scale bars are $1 \mu \mathrm{m}$ ). (right) Intensity oscillation profile of highlighted molecules, showing open and closed super-beacon conformation. E, Schematic representation of super-beacons on a coverslip surface for in vitro characterisation by single-molecule imaging 
pair in a molecule that exhibits two conformational states: "closed"quenched due to fluorophore and quencher contact; "open"fluorescent where the fluorophore and quencher are not in proximity (Figure 1A). ${ }^{26}$ The transition between these conformations thus influences the lifetime of emitting and non-emitting states. We designed a new class of photoswitchable probes using these general principles. We chose a DNA-hairpin scaffold due to its flexibility and easy synthesis, a structure resembling the well-characterised molecularbeacons (Figure 1B). ${ }^{27-30}$ Our probe dubbed a super-beacon (SB), is a single-stranded DNA (ssDNA) oligo-nucleotide with a fluorophore and quencher covalently bound to opposing ends (Figure 1B). Short, complementary, terminal sequences promote self-hybridization resulting in the stable formation of the hairpin-shaped secondary structure, hereafter called the closed-state. This state, dominant in thermal equilibrium, is non-emitting due to resonant energy transfer, collision and contact with a dark quencher. ${ }^{31,32}$ The probe can reversibly transition to a short-lived fluorescent "open" state in a stochastic manner (Figure $1 \mathrm{C}$ ). The rate of transition between states is modulated by temperature, chemical environment and choice of structure (oligonucleotide sequence) ${ }^{33}$ (Figure $1 \mathrm{C}$ ). To demonstrate the principles of SBs, we designed a short probe with the sequence 5'-ATTO550 ACG TTT T[Biotin-dT]T TTT CGT BHQ2-3' called B3S9L-ATTO550-BHQ2. A naming convention that relates to it being a super-Beacon (B3S9L) using 3 base pairs in the Stem region (B3S9L) and 9 poly-T bases in the Loop (B3S9L), an ATTO550 fluorophore conjugated to $5^{\prime}$-end and a Black Hole Quencher 2 (BHQ2) conjugated to $3^{\prime}$-end. A control structure without quencher was designed 5'-ATTO550 ACG TTT T [Biotin-dT]T TTT CGT $-3^{\prime}$. Both probes have an internal modification to the thymine base at the centre of the hairpin loop to biotinylated thymine. This feature allows their binding to streptavidin-conjugated labelling agents such as antibodies or treated coverslips for optical characterisation (Figure 1D,E).

\section{2 | Super-beacons in vitro characterisation}

To characterise SBs as photoswitching probes, we imaged their photophysical behaviour while immobilised on a coverslip using Total Internal Reflection Fluorescence microscopy (TIRF) (Figure 1D,E). B3S9L-ATTO550-BHQ2 SBs were linked to a coverslip surface at high dilution, to achieve sparse spatial distribution and negligible detectable molecular spatial overlap. In SMLM, it is expected that probes switch stochastically between a non-fluorescent (off) state and fluorescent (on) state. The on-state lifetime, $\tau_{\mathrm{ON}}$, should, ideally, be similar to the acquisition rate (10 s of milliseconds) and ratio of onstate to off-state lifetimes, $r=\tau_{\mathrm{ON}} / \tau_{\mathrm{OFF}}$, at least $1 / 50 .{ }^{33}$ Following the protocols described in the Methods section, we measured the switching state lifetimes of our SB structure (Figure 2A). In these conditions, $r=1 / 50$ is achieved at $250 \mathrm{~W} / \mathrm{cm}^{2}$, demonstrating desirable photoswitching at 5 -fold less illumination than for our control probe. Interestingly, we observed an illumination dependence on the on- to off-state lifetimes ratio. This can be due to local thermal effects or due to the photophysics of the ATTO555 fluorophore, namely the transitions to triplet states at these illumination regimes. To explore this effect further we designed new SB, where we replaced the ATTO555 fluorophore for the much less photoswitching prone ATTO647N (5'-ATTO647N ACG TTT T[Biotin-dT]T TTT CGT BHQ2-3' called B3S9L-ATTO647N-BHQ2). Owing to the photophysical characteristics of ATTO647N we observed much less illumination dependency in the ratio of on- to off-state lifetimes (Figure S1A), an effect that we could recover by imaging this SB in OxEA switching buffer (Figure S1A). ${ }^{34}$

Additionally, the molecular design of SBs favours the closed-state conformation, an advantage over organic fluorophores for SMLM, since a high proportion are observed to be in the off-state at the beginning of acquisition (Figure 2B). We then disrupted the hairpin structure with formamide, to determine if switching properties are the result of the transition between conformation states. The delayed addition of formamide and consequent melting of the hairpin structure induced an on-state indistinguishable intensity from the control structure (Figure $2 \mathrm{C}$ ). To further explore the structural, chemical and thermal tunability of SBs we designed a SB with a larger stem (5'ATTO647N CGCTC TTT TTT TTT [Biotin-dT] TTT TTT TTT GAGCG BHQ2-3', called B5S19L-ATTO647N-BHQ2). The longer stem region should provide extra stability between fluorophore and quencher, and, consequently, a lower on- to off-state lifetime ratio when compared with B3S9L. To access the SB structural, chemical and thermal tunability we analysed B3S9L-ATTO647N-BHQ2, B5S19LATTO647N-BHQ2 and the control probe behaviour in a low osmotic media (MiliQ) at $23^{\circ} \mathrm{C}$ and in a higher osmotic media (phosphatebuffered saline, PBS) at $37^{\circ} \mathrm{C}$. If our hypothesis is correct we should obtain the same on- to off-state lifetime ratio in both imaging media for the different probes, as the temperature increase should compensate for the higher osmotic strength of the media. Additionally, in equivalent imaging media (MiliQ or PBS) we should see a decrease in the on- to off-state lifetimes ratio due to the increase in the SB stem - B5S19L<B3S9L. As expected, we observed a similar behaviour between the SB probes in MiliQ water at $23^{\circ} \mathrm{C}$ and PBS at $37^{\circ} \mathrm{C}$, and we see a clear shift for lower ratio of on- to off-state lifetimes in B5S19L-ATTO647N-BHQ2 comparing to B3S9L-ATTO647N-BHQ2 (Figure S1B,C).

\section{3 | Super-beacons as super-resolution probes}

To evaluate the potential of SBs as imaging probes, we took advantage of the biotin-modified thymine in the SB loop to attach it to a streptavidin-conjugated antibody (Figure 2D,E). Using these antibodies we labelled and imaged $\beta$-tubulin in fixed NIH3T3 cells (MetOH fixed as previously described ${ }^{11}$ ) with an illumination intensity of $150 \mathrm{~W} / \mathrm{cm}^{2}$ in PBS buffer. These low illumination and non-toxic buffer conditions are within the range of classical widefield live-cell imaging experiments. ${ }^{15}$ Figure 2D shows SRM imaging with both the B3S9L-ATTO550-BHQ2 probe and B3S9L-ATTO550 control probe. SMLM analysis was performed using ThunderSTORM ${ }^{35}$ detecting over $1 \times 10^{6}$ localizations (full-frame) and generating a 

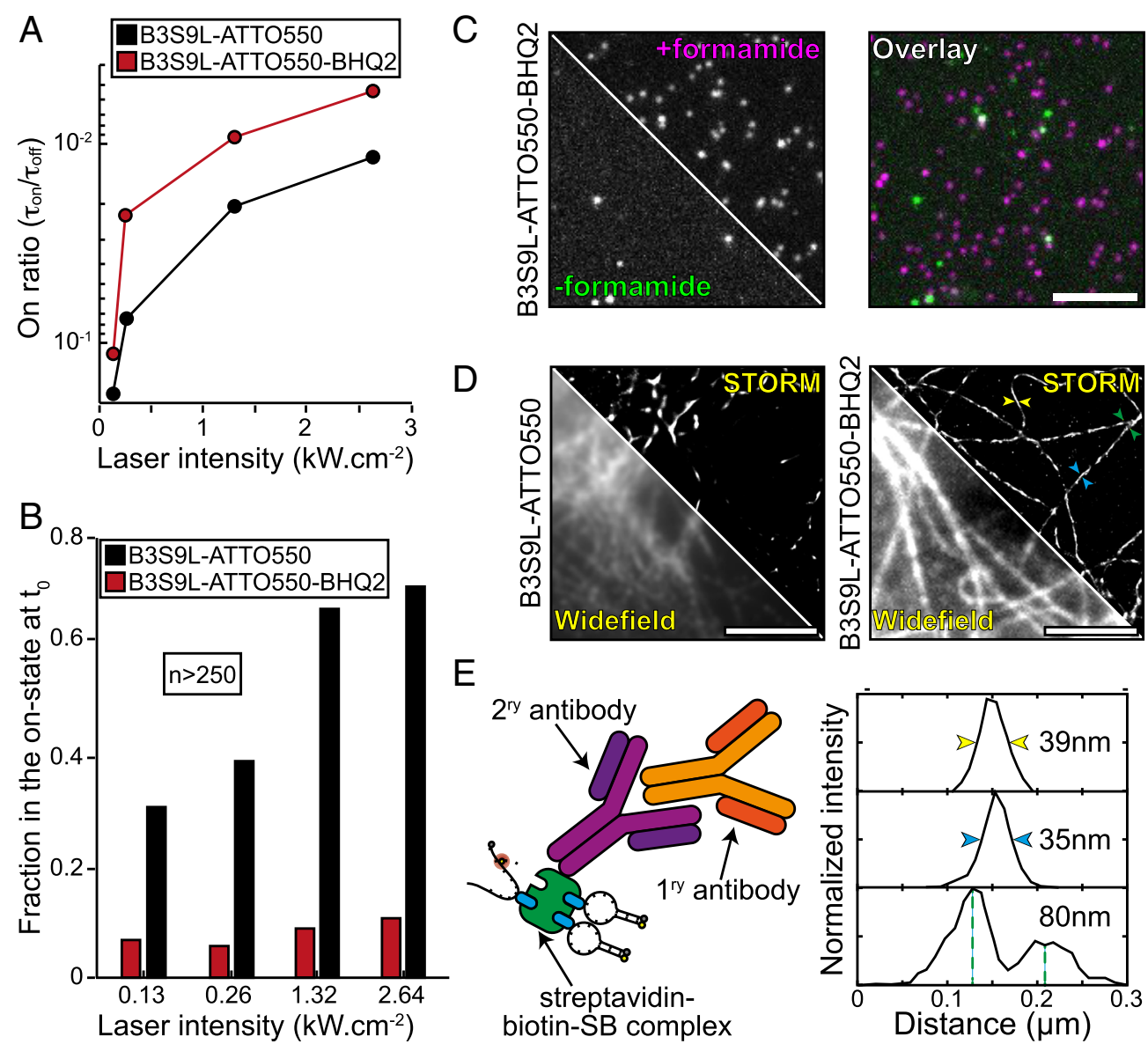

FIGURE 2 Super-beacons characterisation as SMLM probes. A, On time ratio $r=\tau_{\mathrm{ON}} / \tau_{\mathrm{OFF}}$, against illumination intensity, where $\tau_{\mathrm{ON}}$ and $\tau_{\mathrm{OFF}}$ are the weighted means of the on- and dark-state distributions respectively. (Red line) B3S9L-ATTO550-BHQ2. (Black line) B3S9L-ATTO550. B, Fraction of super-beacon and control probes in the on-state in the first frame of acquisition. C, Super-beacon probe imaged in the absence (-formamide) or presence (+formamide) of formamide and overlay of both images (overlay). D, Pre-acquisition snapshot (WF) and SMLM reconstruction (STORM) of $\beta$-tubulin immunolabelling with B3S9L SB (B3S9L-ATTO555-BHQ2) and control probe (B3S9L-ATTO555). Scale bars are $5 \mu \mathrm{m}$. E, Schematic representation of the strategy used to perform $\beta$-tubulin immunolabelling (left), measurement of Full With Half Maximum (FWHM) and distances between structures on the highlighted regions in D

super-resolution reconstruction with improved contrast and resolution in comparison to the equivalent widefield image (Figure 2D). A low-density region of the microtubule network was selected to judge image quality and resolution. SB-based $\beta$-tubulin SRM images show good image fidelity comparing to the WF image, with an average spatial resolution of $50 \mathrm{~nm}$, as seen by Fourier Ring Correlation (FRC), calculated using the NanoJ-SQUIRREL ImageJ plugin ${ }^{36}$ (Figure S2). This was confirmed by measuring the distance between two filaments (Figure 2D,E, suggesting a resolution better than $80 \mathrm{~nm}$ ). This experiment demonstrates that for similar low-illumination imaging conditions, using a non-toxic buffer, SBs can achieve SRM images of higher perceived quality than those obtained with the control probe (Figure 2D).

\subsection{Capacity for live-cell SRM}

To test the potential of SBs for live-cell SRM imaging, we used cell surface-expressed interferon-induced transmembrane (IFITM) proteins. IFITM proteins are a family of broad-spectrum inhibitors of virus replication that act primarily against enveloped viruses. ${ }^{37}$ IFITM genes have been found in numerous vertebrate species. In humans, IFITM-1, -2 and -3 encode proteins that are thought to act primarily, though perhaps not exclusively, ${ }^{38}$ by inhibiting viral fusion. ${ }^{37,39}$ When expressed alone in A549 cells, IFITM-1 localises predominantly to the plasma membrane, while IFITM-2 and -3 localise preferentially in late and early endosomes, respectively. ${ }^{39}$ Thus, for human IFITM proteins at least, the distributions cover the main cellular portals through which enveloped viruses enter cells. We next investigated the potential of SBs to provide detailed information on the distribution and dynamics of C-terminally HA-tagged IFITM-1 in live-cell SMLM experiments. As IFITM- 1 is primarily localised to the plasma membrane, and its $C$ terminus is accessible on the surface of intact cells, ${ }^{39}$ we chose this protein for initial studies. To first validate IFITM-1 SRM, we performed a fixed-cell comparison between standard STORM with AlexaFluor647 and SB-based STORM. A549 cells stably expressing HA-tagged IFITM-1 were grown on glass coverslips, fixed and labelled either with (a) an anti-HA antibody conjugated to AlexaFluor647 and 
subsequently embedded into a photoswitching inducing buffer (100 mM $\beta$-mercaptoethylamine) or (b) the same anti-HA antibody SBlabelled through conjugation with streptavidin (as mentioned in Figure 2) and embedded in PBS. Both samples were then imaged through several STORM acquisitions where, for each, the illumination intensity was increased from live-cell compatible $50 \mathrm{~W} / \mathrm{cm}^{2}$ to classical STORM illumination intensities $2.5 \mathrm{~kW} / \mathrm{cm}^{2}$ (Figure 3). Despite the order of magnitude difference in illumination intensity across modalities and the SB sample not containing a buffer promoting photoswitching, there were negligible visual differences in IFITM1 distribution at the cell surface, for both imaging modalities (Figure $3 \mathrm{~A}$ ). Quantitative analysis of image quality through the SQUIRREL algorithm $^{36,40}$ further shows that both conditions achieve similar quality (Figure S3). In all illumination regimes, there was a high fidelity between the SMLM reconstructions and the raw data (as seen by SQUIRREL error maps). As expected, this was not the case for AF647 that relies on photo-induced processes (Figure S3A,B). SMLM provides not only high-resolution structural information but also quantitative information about the subcellular distribution and molecular organisation. ${ }^{41}$ Hence, we asked if the high fidelity in protein distribution across different illumination intensity regimes was translated into the ability to extract quantitative data. To access this, we analysed IFITM1 distribution across a range of illumination intensities (from $\sim 0.05$ to $\sim 2.5 \mathrm{~kW} / \mathrm{cm}^{2}$ ) and analysed the cluster diameter with SR-Tesseler. ${ }^{41}$ When using anti-HA-AF647 labelled antibody, we saw a significant difference $(P<.01)$ between the high-illumination regime $\left(2.3 \mathrm{~kW} / \mathrm{cm}^{2}\right)$ and the low illumination regimes $\left(>0.46 \mathrm{~kW} / \mathrm{cm}^{2}\right)$ and a corresponding increase in the mean cluster diameter (from $70 \mathrm{~nm}$ to values ranging between 80 and $100 \mathrm{~nm}$ ). For the anti-HA-streptavidin-SB Ab conjugate, we obtained an average cluster diameter of $80 \mathrm{~nm}$. Importantly, we saw no difference $(P>.1)$ between the different illumination regimes. The $10 \mathrm{~nm}$ discrepancies in size between both approaches are indicative of linker length between the fluorophore and the antibody (NHS for AF647 vs streptavidin-biotin for SB). Adjusting the illumination intensity affected the measured cluster diameter when using AlexFluor647, due to the photo-physical dependence of the switching rates, whereas there was no difference when using the SB. Having confirmed that SMLM of HA-tagged IFITM1 is possible, live-cell imaging was performed at $0.132 \mathrm{~kW} / \mathrm{cm}^{2}, 561 \mathrm{~nm}$ illumination with exposure time $20 \mathrm{~ms}$ in Ringers solution (Figure 4). To do this A549 (IFITM1-HA) were incubated with SB labelled anti-HA primary antibody for 30 minutes, at $37^{\circ} \mathrm{C}$ in Ringers solution (cells were washed twice with Ringers solution before imaging, cells were imaged in Ringers solution at $37^{\circ} \mathrm{C}$ ). There was a small deterioration in the localization precision compared to fixed cell imaging, $\sigma_{\text {loc }}=30.7 \mathrm{~nm}$, which is expected due to the reduced photon count when using shorter exposure times and the motion blurring of the molecule during the exposure. The dynamics of the IFITM1 protein could be visualised by rendering of the localizations with a lookup table indicating time (Figure 4A). The increase in the spatial extent of these clusters in comparison to fixed cells was too large to be accounted for by the decrease in localization precision, which could be suggestive of diffusion of the molecule within confined regions. Thin trails of localizations between these regions were also seen in few instances. Single-molecule tracking was performed using the single-particle tracking plugin for ImageJ, TrackMate ${ }^{40}$ (Figure 4B). Single molecules were identified in the raw images and detections were linked to form tracks using an algorithm based on the linear assignment problem proposed for single-molecule tracking in livecells. ${ }^{40}$ Two-frame gap closure with a maximum gap of $200 \mathrm{~nm}$ was performed. A total of 18667 tracks were detected, with a mean duration of 0.11 second and mean velocity of $2.97 \mu \mathrm{m} / \mathrm{s}$. The tracking results allow a single particle tracking image of the tracks to be generated (Figure 4B). Consequently, SBs are a promising solution
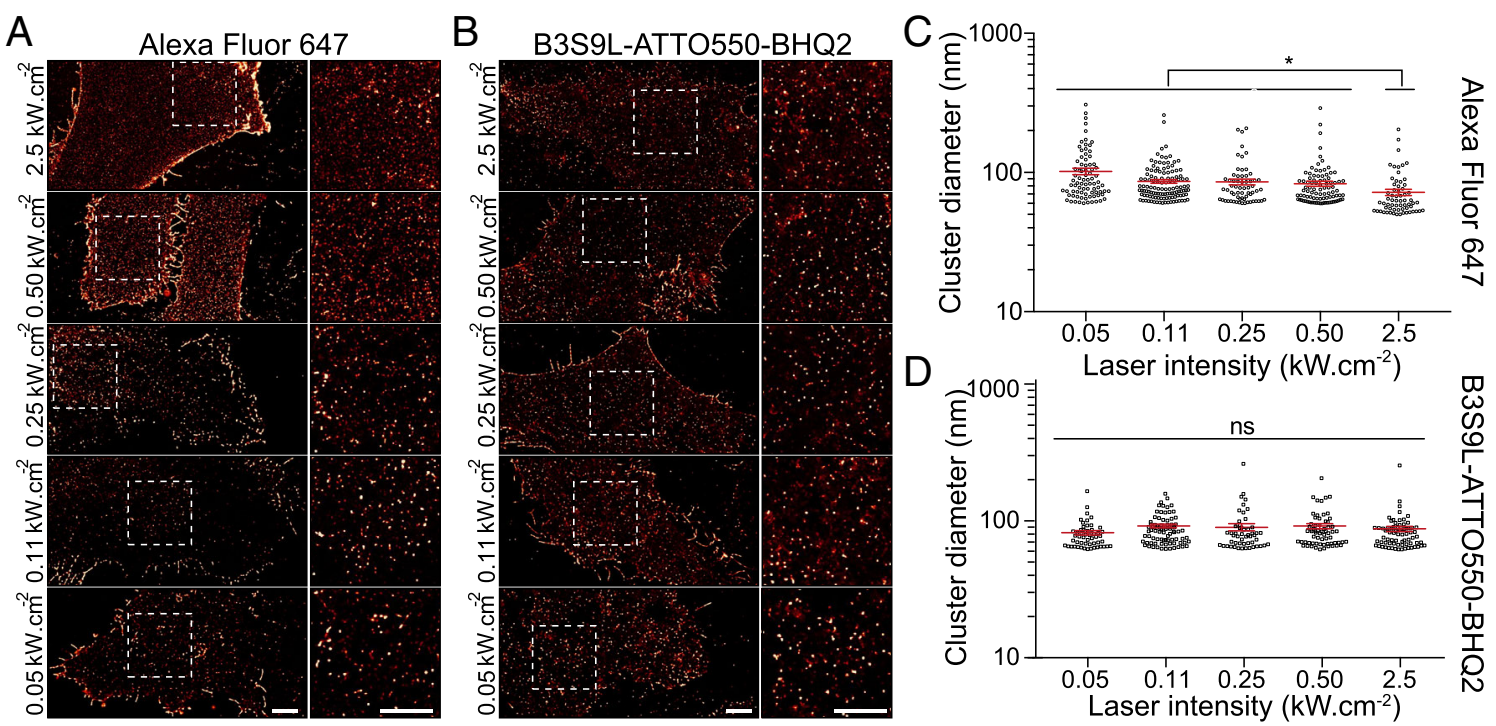

FIGURE 3 SMLM with super-beacons at live-cell compatible illuminations. A, IFITM1 STORM reconstruction using AlexaFluor647 or superbeacon B3S9L conjugated primary antibodies at increasing illumination intensities. Scale bars are $1 \mu \mathrm{m}$. B, SR-Tesseler cluster analysis of IFITM1 cluster diameter $(\mathrm{nm})$ at increasing illumination intensities with anti-HA primary antibodies conjugated with AlexaFluor647 or super-beacon B3S9L 

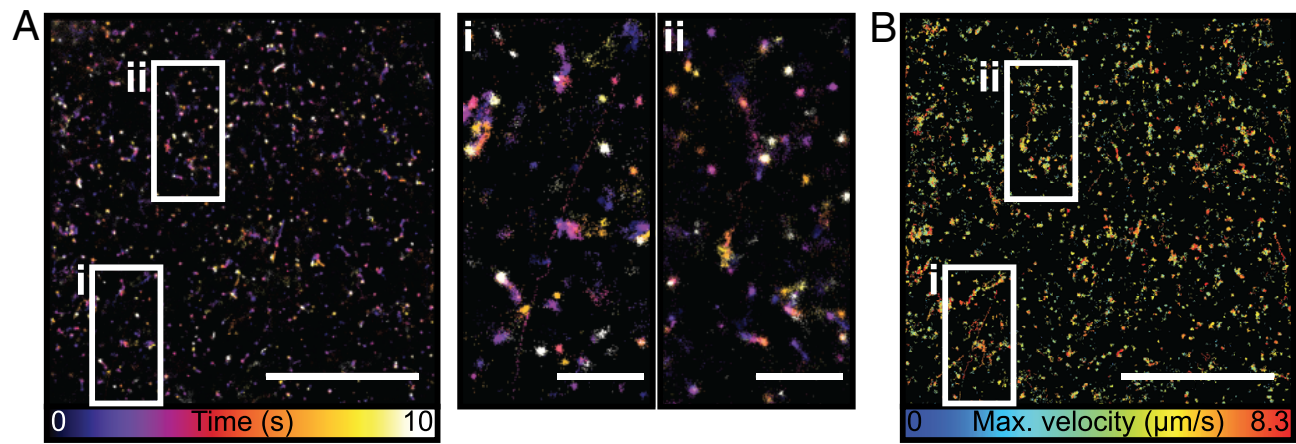

F I G URE 4 Live-cell SR single particle tracking with super-beacons. A, Histogram localizations from the first 10s of acquisition using superbeacon B3S9L, on $20 \mathrm{~nm}$ pixel grid coloured by time of localization. (I and II) Zoomed-in insets. Scale bar, $10 \mu \mathrm{m}$ and $2 \mu \mathrm{m}$ in insets. B, Single molecule tracks identified in a 30000 frame acquisition using the SB in live-cells. Tracks coloured by maximum velocity (highlighted regions are the same as in A). Scale bar, $10 \mu \mathrm{m}$

for the analysis of the diffusion of membrane-bound extracellular proteins on the molecular scale.

\section{3 | CONCLUSION}

Imaging of super-beacon labelled proteins in live-cells in low illumination regimes resulted in single-molecule localizations with precisions $\sim 30 \mathrm{~nm}$ and SMLM reconstructions with sub $100 \mathrm{~nm}$ resolutions, which demonstrates the potential of these probes in super-resolution. The ability to achieve this in a physiological buffer at low-illumination intensities demonstrates the merits of using SBs in live-cell settings. Furthermore, combining SB with techniques such as single-particle tracking has the potential to allow experiment design using SB imaging to answer biologically relevant questions such as, in the example here, how IFITM proteins inhibit viral infection.

Limitations were identified, such as restrictions on labelling density due to steric hindrance and the biological relevance of ssDNA in live-cells. The size of the Ab conjugate hinders the use of SBs for livecell imaging in their current form. The format used in this study, "Ab+ streptavidin + SB", entails a large distance between the probe and the target of interest. This translates in a large linker error visible in the cluster diameter measurements, for example (when compared with Ab directly conjugated to AF647). Other conjugations options are available if the reader wants to use SBs in live-cells. This option requires synthesising SBs with a Thiol modification instead of a biotin modification (also available from Metabion, Thiol-C6), this modification permits following a simple conjugation protocol already commonly used for DNA-PAINT. ${ }^{42}$ Alternatively, if the biotinylated SB solution seems more attractive due to the ease and cost-effective approach of using the same SB for both characterisation and live-cell imaging, monomeric streptavidin is an option. ${ }^{43-45}$ Additionally, reducing the illumination intensity to accommodate photo-toxicity and reducing the exposure time to accommodate dynamics both contribute a reduced number of detected photons. Finally, the duration of the on-states of the SBs presented are long in comparison to the optimal for fast imaging. Due to the nature of the transient quenching and the modularity and flexibility of DNA oligonucleotide design, however, the possibilities for tailoring SBs to specific applications are extensive. For example, a 5 bp stem can have 1024 different sequences. Together with different loop lengths and fluorophores there are thousands of possible 5 bp stem SBs that could be considered. Additionally, the stability of the secondary hairpin structure can be modulated by the design of the oligonucleotide sequence, namely by altering the length of the hairpin and the avidity of the complementary stem region. The hybridization free energy can be determined by the identity of the bases in the stem sequence and experimental conditions such as $\mathrm{pH}$, temperature or ion concentration and can be calculated using standard models of DNA hybridisation. ${ }^{46,47}$ This stability can be further modulated in situ by chemical environment and temperature. These factors combined open the possibility of designing SBs with bespoke properties for specific applications (eg, imaging probes, as showcased here, or sensors) and/or environments (eg, different salt concentrations or temperatures). Some of these properties such as temperature or chemical environment ${ }^{48}$ can be changed dynamically at the microscope to further optimise their photoswitching behaviour while imaging. This flexibility suggests a model-based design principle could be adopted to reach an optimal structure for a specific application in order to maximally make use of the rapid imaging technologies available.

\section{4 | METHODS}

\subsection{Super-beacon probe design}

The SB structure was designed to be short to reduce linker and crowding errors ${ }^{49,50}$ and to have a melting temperature above room temperature $\left(\sim 33^{\circ} \mathrm{C}\right.$-using $\left.\mathrm{mFOLD}{ }^{46}\right)$ to favour the closed-state conformation (see Characterisation section). A SB was designed with the sequence 5'-ATTO550 ACG TTT T[Biotin-dT]T TTT CGT BHQ2-3' called B3S9L-ATTO550-BHQ2, by the naming convention of having 
three base pairs in the Stem and nine poly-T bases in the Loop, an ATTO550 fluorophore conjugated to the $5^{\prime}$ end and a BHQ2 quencher conjugated to the $3^{\prime}$ end (a control structure without the quencher was also designed 5'-ATTO550 ACG TTT T[Biotin-dT]T TTT CGT $-3^{\prime}$ ). Both structures were purchased pre synthesised, purified by high-performance liquid chromatography and lyophilized, from Metabion. Before use SBs were diluted to a concentration of $100 \mu \mathrm{M}$ in TE buffer (10 mM Tris-HCL, $1 \mathrm{mM}$ EDTA, pH 7.5) using the manufactures reported molar quantities and stored at $-20^{\circ} \mathrm{C}$, shielded from light sources. Both probes were designed with an internal modification to the thymine base at the centre of the hairpin loop to a biotinylated thymine to allow the SBs to bind to streptavidin conjugated labelling agents such as antibodies (Ab) or be surface immobilised for in vitro characterisation (Figure 1D,E). For the structural, thermal and chemical analysis of the SB probes (Figure S1), B3S9L-ATTO647N (5'ATTO647N ACG TTT T[Biotin-dT]T TTT CGT-3'), B3S9L-ATTO647NBHQ2 (5'-ATTO647N ACG TTT T[Biotin-dT]T TTT CGT BHQ2-3') and B5S19L-ATTO647N-BHQ2 (5'-ATTO647N CGCTC TTT TTT TTT [Biotin-dT] TTT TTT TTT GAGCG BHQ2-3') were designed, prepared and used as described above.

\section{2 | Surface passivation for single-molecule studies}

In order to determine the photoswitching characteristics of SBs, biotinylated SBs were immobilised on passivated surfaces. To do this $25 \mathrm{~mm}$ coverslips (HiQA-1.5H, SAN 5025-03A, CellPath) were washed as previously described, ${ }^{51}$ followed by Piranha etching. ${ }^{52}$ Cleaned coverslips were amino-silanized and coated with NHS-ester polyethylene glycol (PEG-1-0001, ChemQuest Limited) and NHSester Biotin-PEG (PG2-BNNS-5 k, ChemQuest Limited) as previously described. ${ }^{52}$ Coverslips were washed $10 \times$ with $1 \times$ liquid, sterile-filtered, Dulbecco's Phosphate Buffered Saline plus $5 \mathrm{mM} \mathrm{MgCl}_{2}$ (D8662, Sigma), filtered twice through a $0.22 \mu \mathrm{m}$ filter and stored in a flask under permanent UV bleaching, from here on called "clean PBS". Buffers were UV-bleached (and subsequently stored) under a $370 \mathrm{~nm}$ LED (370 nm LED, LedEngin Inc, LZ1-10UV00-0000), during all steps that are not affect by UV illumination (all steps prior to the addition of streptavidin to the surface). For this we used an open flask lid (eg, 292270 808, DWK Life Sciences), where the 370 nm LED attached to a heatsink (eg, ICK LED R $23.5 \times 14 \mathrm{G}$, Fischer Elektronik) was mounted. Streptavidin was added to coated coverslips at $0.2 \mathrm{mg} / \mathrm{ml}$ in $1 \times$ clean PBS for 10 minutes. Streptavidin-coated coverslips were washed $10 \times$ with $1 \times$ clean PBS. SBs were diluted to a concentration of 10 to $100 \mathrm{nM}$ and coverslips were incubated for 1 to 10 minutes in order to have a low probability that multiple binding sites of streptavidin are occupied by a SB. After SBs were added, $100 \mathrm{~nm}$ TetraSpeck beads (T7279, Life Technologies), for drift correction, were added at a dilution of 1:1000 in 1x clean PBS for 10 minutes to each coverslip. Coverslips were again washed $10 \times$ with $1 \times$ clean PBS. On average number of detections in "empty" coverslips prepared in an identical manner but without the addition of SBs was 0.090 $\pm 0.0092 / \mu \mathrm{m}^{2}$ at $561 \mathrm{~nm}$ illumination, this compares to an average of $0.38 \pm 0.039 / \mu \mathrm{m}^{2}$ for ATTO550 SB-coated coverslips indicating approximately $22 \%$ contribution of spurious noise detection from fluorescent debris in under $561 \mathrm{~nm}$ illumination.

\section{3 | Illumination intensity determination}

The reported illumination intensities are the peak intensities $\left(I_{\text {peak }}\right)$ at the centre of the field of view and were calculated from the percentage of maximum laser power provided by the microscope acquisition software, $p$. For each wavelength used, a conversion factor, $c$, was regularly calculated relating the software provided power to the measured power in the back aperture of the objective, $P=c p$. Over the course of the experiments a maximum of $10 \%$ error was measured. The transmission rate, $T$, of the objective was confirmed by measurement to be $89 \% \pm 1.5 \%$. For each wavelength used, the SD, $\sigma_{l}$, of the Gaussian distributed illumination intensity in the sample plane was measured by fitting the intensity of line profiles taken from illuminated empty coverslips. The calculation for $I_{\text {peak }}$, assuming a Gaussian distributed illumination, $I(x, y)$, of the sample, can be derived as follows,

$$
\begin{gathered}
P . T=\iint_{-\infty}^{+\infty} I(x, y) \mathrm{d} x \mathrm{~d} y+I_{\text {peak }} \cdot 2 \pi \sigma_{I}^{2} \\
I_{\text {peak }}=\frac{c p T}{2 \pi \sigma_{I}^{2}}
\end{gathered}
$$

The maximum variation observed of the illumination intensity over the $5.2 \mu \mathrm{m}$ imaged area was less than $5 \%$.

\section{4 | Single-molecule imaging of super-beacon probes}

Characterisation was performed in phosphate buffer saline (PBS, MiliQ water or OxEA-the latter as previously described ${ }^{34}$-at 23 or $37^{\circ} \mathrm{C}$ ), using a range of illumination intensities, from live-cell compatible, $0.132 \mathrm{~kW} / \mathrm{cm}^{2}$, to classical STORM intensities $2.64 \mathrm{~kW} / \mathrm{cm}^{2}$ for $561 \mathrm{~nm}$ and 0.1 to $0.8 \mathrm{~kW} / \mathrm{cm}^{2}$ for the $647 \mathrm{~nm}$ laser to determine the photoswitching properties of the SB probes. This was achieved through TIRF imaging of prepared coverslips using AttoFluor chambers (A7816, Thermo Fisher Scientific) in an ElyraPS.1 inverted microscope at room temperature $\left(22^{\circ} \mathrm{C}\right)$. For excitation of ATTO550 a $561 \mathrm{~nm}$ laser operating at various illumination intensities was used. A 100x TIRF objective (Plan-APOCHROMAT 100x/1.46 Oil, Zeiss) was used, with additional $1.6 \times$ magnification, to collect fluorescence onto an EMCCD camera (iXon Ultra 897, Andor), yielding a pixel size of $100 \mathrm{~nm}$. Imaging of ATTO550 SBs was performed with an exposure time of $18 \mathrm{~ms}$ using a cropped area of the camera $\left(51.2 \times 25.6 \mu \mathrm{m}^{2}\right)$ continuously for a period of 540 seconds (30 000 frames). 


\section{5 | Single-molecule time-trace analysis}

Data obtained were analysed by extracting intensity over time traces (eg, Figure 1D) using a custom analysis pipeline as follows: Raw data was loaded with ImageJ/Fiji ${ }^{53}$ and single molecule localization performed using the ThunderSTORM plugin. ${ }^{35}$ ThunderSTORM parameters were as follows: Filtering of the data was performed using a BSpline Wavelet filter with scale 2, order 3. Detection of molecules was based on local maximum identification using 8-pixel neighbourhood connectivity. Detection threshold was set to 2 times the SD of the first level of the wavelet decomposition. An integrated Gaussian PSF was used for localisation with initial $\sigma=160 \mathrm{~nm}$. Fitting was performed using a weighted least squares estimate. Following localization of single molecules Thunderstorm was also used to calculate a per frame drift on the basis of fiducial marker localization's using default settings. ThunderSTORM localization results, drift table and corresponding raw image data were loaded by a custom written MATLAB (Math-Works, Inc.) analysis program Results were filtered, retaining localisations with $\sigma_{\mathrm{PSF}}>80 \mathrm{~nm}, \sigma_{\mathrm{PSF}}<200 \mathrm{~nm}, \sigma_{\mathrm{LOC}}<40 \mathrm{~nm}$ and $I>40$ photons, where $\sigma_{\mathrm{PSF}}$ is the fitted SD of the Gaussian PSF, $\sigma_{\text {LOC }}$ is the ThunderSTORM calculated precision and $I$ is the ThunderSTORM calculated photon count. This removes spurious fits to single pixels with narrow $\sigma_{\mathrm{PSF}}$, which are common in ThunderSTORM analysis, and low precision and low photon count localizations. The drift corrected, filtered localizations were subsequently grouped using a frame by frame search for localizations separated by less than $100 \mathrm{~nm}$ from the continuously updated mean position of the grouped localizations. Groups with mean positions separated by less than $100 \mathrm{~nm}$ following the full search were merged. Any groups of localizations with a number of members greater than $75 \%$ of the total number of frames were identified as fiducial markers and any groups within a radius of $2 \mu \mathrm{m}$ of these fiducial markers were removed from the localization list. Localizations were clustered using DBSCAN ${ }^{54}$ with a minimum number of cluster members, minPts $=2$, and neighbourhood radius, $\varepsilon=80 \mathrm{~nm}$ ( $2 \times$ the maximum expected localization error after filtering). The cluster members were used to determine the average position of single molecules and any single molecules separated by less than $200 \mathrm{~nm}$ were discarded. The remaining molecule positions were used to extract $5 \times 5$ pixel $\left(0.25 \mu \mathrm{m}^{2}\right)$ regions centred (without interpolation) on the molecule position from the drift corrected (without interpolation) raw data to produce a set of "trace images". Any molecules that drifted into or out of the field of view during acquisition were discarded. The median of each full frame of the raw data was subtracted from the trace image frames. Due to the sparsity of the molecule detections this accounted for the contribution of illumination intensity variation to the background signal (approximately $2 \%$ across the time of acquisition). The background intensity of each frame of the trace images was calculated as the mean of the 16 boundary pixels and subtracted from the trace images. The photon conversion factor of the EMCCD camera was used to convert background subtracted camera counts in the trace images to photon number trace images. The photon number trace images were converted to photon number per frame traces by integration of the convolution of each trace image with a correctly normalised Gaussian PSF. The photon number per frame traces were subsequently converted to SNR per frame traces by division by the SD of all boundary pixels in the convolved trace image sequence. Denoising of the SNR traces was performed in an automated manner by thresholding of the detail coefficients of a wavelet decomposition of the trace ${ }^{55-57}$ using the "wden" function in MATLAB. Briefly, Wavelet decomposition of the 1D SNR trace was performed using a 6 level, Haar-Wavelet. Thresholds for each level are determined on a heuristic basis using Stein's unbiased risk principle where the risk function has high SNR and a fixed form universal threshold $\sigma \sqrt{2 \ln N}$ otherwise, where $N$ is the dataset length. ${ }^{58}$ Thresholds are scaled by a level dependant estimation of the level noise, $\sigma_{\text {lev }}$, in order to account for non-white noise. The detail coefficients are soft-thresholded and the denoised trace is computed from the wavelet reconstruction using the original approximation coefficients of level 6 and the thresholded detail coefficients of levels 1 to 6 . The denoised SNR trace is hard thresholded at $10 \times$ the SD of SNR values $<2$ to determine on- or dark-state identities for each frame. Finally, transition times and state lifetime distributions were aggregated from the resulting idealised on/dark traces. The initial state was recorded separately in order to form initial on-state and initial dark-state distributions and the last state of each trace was discarded as corresponding to either an on-state truncated by the end of acquisition or an off state that is indistinguishable from a bleached state.

\subsection{Super-beacon antibody conjugation}

An anti-mouse secondary antibody (A21236-Thermo Fisher Scientific) was conjugated to streptavidin using the Lightning-Link Streptavidin kit (708-0030, Innova Biosciences-following the manufacturer recommendations). After conjugation the StreptavidinAntibody conjugate was incubated with $100 \mathrm{nM}$ B3S9LATTO550-BHQ2-biotin SB or $100 \mathrm{nM}$ B3S9L-ATTO550-biotin control for 16 hours at $4 C$ on a shaker in the dark. The resulting SB labelled $\mathrm{Ab}$ was purified via $100 \mathrm{kDa}$ amicon spin filters (Merckfollowing the manufacturer recommendations).

\section{7 | Super-beacon for fixed cell imaging of microtubules}

Fixed NIH3T3 cells were stained for $\beta$-tubulin (as previously described $^{11}$ ) with SB (B3S9L-ATTO550-BHQ2), and control probe without quencher (B3S9L-ATTO550), conjugated secondary Abs. These samples were imaged with an illumination intensity of $0.132 \mathrm{~kW} / \mathrm{cm}^{2}$ in PBS. The SMLM analysis of the SB labelled $\beta$-tubulin in fixed NIH3T3 cells data was performed using ThunderSTORM generating over $1 \times 10^{6}$ localizations with a mean precision $\left(\sigma_{\text {loc }}\right)$ of $22.0 \mathrm{~nm}$ (estimated by ThunderSTORM ${ }^{35}$ ). A low density region of the microtubule network was selected to judge the maximal achieved quality of the super-resolution image by calculating the full 
width half maximum (FWHM) on two representative line profiles perpendicular to microtubules. Because line profiles of microtubules can be narrowed by artefacts in localization algorithms, ${ }^{58}$ the limit of discrimination of the separation of two crossing microtubules was also measured. This analysis was complemented with NanoJ-SQUIRREL ${ }^{36}$ error mapping and FRC determination (Figure S2).

\section{8 | Super-beacon vs AF647 conjugated antibodies for fixed cell imaging of IFITM1}

We imaged fixed cells (4\% PFA at $23^{\circ} \mathrm{C}$ for 30 minutes) stably expressing HA-tagged IFITM1 A549 cells stained with SBs (anti-HA Ab conjugated with streptavidin as previously mentioned) or AF647 (NHS ester conjugated) at increasing laser powers, from live-cell friendly $\sim 0.05 \mathrm{~kW} / \mathrm{cm}^{2}$ to classical STORM illumination intensities $\sim 2.5 \mathrm{~kW} / \mathrm{cm}^{2}$, in physiological PBS and toxic $\beta$-mercaptoethylamine (MEA), respectively. The SMLM analysis of the data was performed using ThunderSTORM ${ }^{35}$ and to access the fidelity between the SMLM reconstructions and the raw data we used SQUIRREL. ${ }^{36}$ To analyse IFITM1 distribution across a range of illumination intensities (from $\sim 0.05$ to $\sim 2.5 \mathrm{~kW} / \mathrm{cm}^{2}$ ) we analysed the cluster diameter with SR-Tesseler. ${ }^{41}$ Localization tables from ThunderSTORM were imported and Voronoi diagrams were created. Regions of interest were selected with a density factor $\delta$ of 2 . Within individual objects, clusters were identified with $\delta=2$. Statistical analysis was performed using GraphPad Prism 7 (Prism Software). Significance was calculated using unpaired $t$-tests: ${ }^{*} P<.01$.

\section{9 | Super-beacon SR single particle tracking of IFITM1 in live cells}

A549 (IFITM1-HA) were incubated with SB labelled Anti-HA primary antibody for 30 minutes, at $37^{\circ} \mathrm{C}$ in Ringers solution (cells were washed twice with Ringers solution before imaging). Live-cell imaging was performed at $0.132 \mathrm{~kW} / \mathrm{cm}^{2}, 561 \mathrm{~nm}$ illumination with exposure time $20 \mathrm{~ms}$ in Ringers at $37^{\circ} \mathrm{C}$. Single molecule tracking was performed using the single particle tracking plugin for ImageJ, TrackMate. ${ }^{40}$ Single molecules were identified in the raw images and detections were linked to form tracks using an algorithm based on the linear assignment problem proposed for single molecule tracking in live-cells. ${ }^{40}$ Two frame gap closure with a maximum gap of $200 \mathrm{~nm}$ was performed. A total of 18667 tracks were detected, with mean duration of 0.11 second and mean velocity of $2.97 \mu \mathrm{m} / \mathrm{s}$.

\section{ACKNOWLEDGMENTS}

This work was funded by grants from the UK eBiotechnology and Biological Sciences Research Council (BB/M022374/1; BB/P027431/1; BB/R000697/1) (R.H., P.M.P.), the UK Medical Research Council (MR/K015826/1) (R.H.), the Wellcome Trust (203276/Z/16/Z) (R.H.). N.G. was supported by a PhD fellowship from the Engineering and Physical Sciences Research Council (EP/L504889/1). We would like to thank
Dr. Siân Culley and Dr. David Albrecht (University College London) for critical reading and advice in the preparation of this manuscript.

\section{CONFLICT OF INTEREST}

The authors declare no competing financial interests.

\section{AUTHOR CONTRIBUTIONS}

P.M.P., N.G. and R.H. devised the experiments. P.M.P. and N.G. acquired the experimental data sets and analysed the data. M.M.M. and M.M. provided research advice and reagents. The paper was written by P.M.P., N.G. and R.H. with editing contributions of all the authors.

\section{REFERENCES}

1. Rino J, Braga J, Henriques R, Carmo-Fonseca M. Frontiers in fluorescence microscopy. Int J Dev Biol. 2009;53(8-9-10):1569-1579. https://doi.org/10.1387/ijdb.072351jr.

2. Schlichthaerle T, Eklund A, Schueder F, et al. Site-specific labeling of affimers for DNA-PAINT microscopy. Angew Chem Int Ed Engl. 2018; 57:11060-11063. https://doi.org/10.1002/anie.201804020.

3. Tosheva KL, Yuan Y, Matos Pereira P, Culley S, Henriques R. Between life and death: strategies to reduce phototoxicity in super-resolution microscopy. J Phys D Appl Phys. 2020;53(16):163001. https://doi.org/ 10.1088/1361-6463/ab6b95.

4. Herbert S, Soares H, Zimmer C, Henriques R. Single-molecule localization super-resolution microscopy: deeper and faster. Microsc Microanal. 2012;18(6):1419-1429. https://doi.org/10.1017/S1431927612013347.

5. Almada P, Culley S, Henriques R. PALM and STORM: into large fields and high-throughput microscopy with sCMOS detectors. Methods. 2015;88:109-121. https://doi.org/10.1016/j.ymeth.2015.06.004.

6. Betzig E, Patterson GH, Sougrat R, et al. Imaging intracellular fluorescent proteins at nanometer resolution (SOM). Science. 2006;313: 1642-1646. https://doi.org/10.1126/science.1127344.

7. Hess ST, Girirajan TPK, Mason MD. Ultra-high resolution imaging by fluorescence photoactivation localization microscopy. Biophys J. 2006;91(11):4258-4272. https://doi.org/10.1529/BIOPHYSJ.106. 091116.

8. Rust MJ, Bates M, Zhuang X. Sub-diffraction-limit imaging by stochastic optical reconstruction microscopy (STORM). Nat Methods. 2006;3 (10):793-795. https://doi.org/10.1038/nmeth929.

9. Heilemann $M$, van de Linde $S$, Schüttpelz $M$, et al. Subdiffractionresolution fluorescence imaging with conventional fluorescent probes. Angew Chem Int Ed Engl. 2008;47(33):6172-6176. https://doi. org/10.1002/anie.200802376.

10. Dertinger T, Colyer R, lyer G, Weiss S, Enderlein J. Fast, backgroundfree, 3D super-resolution optical fluctuation imaging (SOFI). Proc Natl Acad Sci USA. 2009;106(52):22287-22292. https://doi.org/10.1073/ pnas.0907866106.

11. Gustafsson N, Culley S, Ashdown G, Owen DM, Pereira PM, Henriques R. Fast live-cell conventional fluorophore nanoscopy with ImageJ through super-resolution radial fluctuations. Nat Commun. 2016;7:12471. https://doi.org/10.1038/ncomms12471.

12. Culley S, Tosheva KL, Matos Pereira P, Henriques R. SRRF: Universal live-cell super-resolution microscopy. Int J Biochem Cell Biol. 2018; 101:74-79. https://doi.org/10.1016/j.biocel.2018.05.014.

13. Sage D, Pham T-A, Babcock $H$, et al. Super-resolution fight club: assessment of 2D and 3D single-molecule localization microscopy software. Nat Methods. 2019;16(5):387-395. https://doi.org/10. 1038/s41592-019-0364-4.

14. Patel L, Gustafsson N, Lin Y, Ober R, Henriques R, Cohen E. A hidden markov model approach to characterizing the photo-switching 
behavior of fluorophores. Ann Appl Stat. 2019;13(3):1397-1429. https://doi.org/10.1214/19-AOAS1240.

15. Wäldchen S, Lehmann J, Klein T, van de Linde S, Sauer M. Lightinduced cell damage in live-cell super-resolution microscopy. Sci Rep. 2015;5:15348. https://doi.org/10.1038/srep15348.

16. Henriques R, Griffiths C, Hesper Rego E, Mhlanga MM. PALM and STORM: unlocking live-cell super-resolution. Biopolymers. 2011;95(5): 322-331. https://doi.org/10.1002/bip.21586.

17. Brakemann T, Stiel AC, Weber G, et al. A reversibly photoswitchable GFP-like protein with fluorescence excitation decoupled from switching. Nat Biotechnol. 2011;29(10):942-950. https://doi.org/10. 1038/nbt.1952.

18. Zhang X, Chen X, Zeng Z, et al. Development of a reversibly switchable fluorescent protein for super-resolution optical fluctuation imaging (SOFI). ACS Nano. 2015;9(3):2659-2667. https://doi.org/10. 1021/nn5064387.

19. Arai $\mathrm{Y}$, Takauchi $\mathrm{H}$, Ogami $\mathrm{Y}$, et al. Spontaneously blinking fluorescent protein for simple single laser super-resolution live cell imaging. ACS Chem Biol. 2018;13:1938-1943. https://doi.org/10.1021/acschembio.8b00200.

20. Jungmann R, Steinhauer C, Scheible M, Kuzyk A, Tinnefeld P, Simmel FC. Single-molecule kinetics and super-resolution microscopy by fluorescence imaging of transient binding on DNA Origami_SI. Nano Lett. 2010;10(11):4756-4761. https://doi.org/10.1021/ nl103427w.

21. Jungmann R, Avendaño MS, Woehrstein JB, Dai M, Shih WM, Yin P. Multiplexed 3D cellular super-resolution imaging with DNA-PAINT and Exchange-PAINT. Nat Methods. 2014;11(3):313-318. https://doi. org/10.1038/nmeth.2835.

22. Sharonov A, Hochstrasser RM. Wide-field subdiffraction imaging by accumulated binding of diffusing probes. Proc Natl Acad Sci. 2006; 103(50):18911-18916. https://doi.org/10.1073/pnas.0609643104.

23. Schueder F, Strauss MT, Hoerl D, et al. Universal super-resolution multiplexing by DNA exchange. Angew Chemie-Int Ed. 2017;56(14): 4052-4055. https://doi.org/10.1002/anie.201611729.

24. Auer A, Strauss MT, Schlichthaerle T, Jungmann R. Fast, backgroundfree DNA-PAINT imaging using FRET-based probes. Nano Lett. 2017 17(10):6428-6434. https://doi.org/10.1021/acs.nanolett.7b03425.

25. Tyagi S, Kramer FR. Molecular beacons: probes that fluoresce upon hybridization. Nat Biotechnol. 1996;14(3):303-308. https://doi.org/ 10.1038/nbt0396-303.

26. Doose $\mathrm{S}$, Neuweiler H, Sauer M. Fluorescence quenching by photoinduced electron transfer: a reporter for conformational dynamics of macromolecules. ChemPhysChem. 2009;10(9-10):1389-1398. https://doi.org/10.1002/cphc.200900238.

27. Barreiro LB, Henriques R, Mhlanga MM. High-throughput SNP genotyping: combining tag SNPs and molecular beacons. Methods $\mathrm{Mol}$ Biol. 2009;578:255-276. https://doi.org/10.1007/978-1-60327-4111_17.

28. Tyagi S, Marras SA, Kramer FR. Wavelength-shifting molecular beacons. Nat Biotechnol. 2000;18(11):1191-1196. https://doi.org/10. 1038/81192

29. Tan W, Wang K, Drake TJ. Molecular beacons. Curr Opin Chem Biol. 2004;8(5):547-553. https://doi.org/10.1016/j.cbpa.2004.08.010.

30. Fang X, Li JJ, Perlette J, Tan W, Wang K. Molecular beacons: novel fluorescent probes. Anal Chem. 2000;72(23):747A-753A. https://doi. org/10.1021/ac003001i.

31. Marras SAE. Interactive fluorophore and quencher pairs for labeling fluorescent nucleic acid hybridization probes. Mol Biotechnol. 2008;38 (3):247-255. https://doi.org/10.1007/s12033-007-9012-9.

32. Marras SAE. Selection of fluorophore and quencher pairs for fluorescent nucleic acid hybridization probes. Methods Mol Biol. 2006;335:316. https://doi.org/10.1385/1-59745-069-3:3.

33. Stiehl $\mathrm{O}$, Weidner-Hertrampf $\mathrm{K}$, Weiss $\mathrm{M}$. Kinetics of conformational fluctuations in DNA hairpin-loops in crowded fluids. New J Phys.
2013;15(11):113010. https://doi.org/10.1088/1367-2630/15/11/ 113010 .

34. Nahidiazar L, Agronskaia AV, Broertjes J, Van Broek B, Den JK. Optimizing imaging conditions for demanding multi-color super resolution localization microscopy. PLoS One. 2016;11(7):1-18. https://doi.org/ 10.1371/journal.pone.0158884.

35. Ovesný M, Kř́žek P, Borkovec J, Švindrych Z, Hagen GM. ThunderSTORM: a comprehensive ImageJ plug-in for PALM and STORM data analysis and super-resolution imaging. Bioinformatics. 2014;30(16): 2389-2390. https://doi.org/10.1093/bioinformatics/btu202.

36. Culley $\mathrm{S}$, Albrecht $\mathrm{D}$, Jacobs $\mathrm{C}$, et al. Quantitative mapping and minimization of super-resolution optical imaging artifacts. Nat Methods. 2018;15(4):263-266. https://doi.org/10.1038/nmeth.4605.

37. Smith S, Weston S, Kellam P, Marsh M. IFITM proteins-cellular inhibitors of viral entry. Curr Opin Virol. 2014;4:71-77. https://doi.org/10. 1016/j.coviro.2013.11.004.

38. Lee W-YJ FRM, Liang C, Sloan RD. IFITM proteins inhibit HIV-1 protein synthesis. Sci Rep. 2018;8(1):14551. https://doi.org/10.1038/ s41598-018-32785-5.

39. Weston S, Czieso S, White IJ, et al. Alphavirus restriction by IFITM proteins. Traffic. 2016;17(9):997-1013. https://doi.org/10.1111/tra. 12416.

40. Laine RF, Tosheva KL, Gustafsson N, et al. NanoJ: a high-performance open-source super-resolution microscopy toolbox. J Phys D Appl Phys. 2019;52(16):163001. https://doi.org/10.1088/1361-6463/ab0261.

41. Levet F, Hosy E, Kechkar A, et al. SR-Tesseler: a method to segment and quantify localization-based super-resolution microscopy data. Nat Methods. 2015;12(11):1065-1071. https://doi.org/10.1038/nmeth.3579.

42. Schnitzbauer J, Strauss MT, Schlichthaerle T, Schueder F, Jungmann R. Super-resolution microscopy with DNA-PAINT. Nat Protoc. 2017;12 (6):1198-1228. https://doi.org/10.1038/nprot.2017.024.

43. Howarth M, Ting AY. Imaging proteins in live mammalian cells with biotin ligase and monovalent streptavidin. Nat Protoc. 2008;3(3):534545. https://doi.org/10.1038/nprot.2008.20.

44. Qureshi MH, Yeung JC, Wu SC, Wong SL. Development and characterization of a series of soluble tetrameric and monomeric streptavidin muteins with differential biotin binding affinities. J Biol Chem. 2001; 276(49):46422-46428. https://doi.org/10.1074/jbc.M107398200.

45. Wu S-C, Wong S-L. Engineering soluble monomeric streptavidin with reversible biotin binding capability. J Biol Chem. 2005;280(24):23225 23231. https://doi.org/10.1074/jbc.M501733200.

46. Zuker M. Mfold web server for nucleic acid folding and hybridization prediction. Nucleic Acids Res. 2003;31(13):3406-3415. https://doi. org/10.1093/nar/gkg595.

47. SantaLucia J. A unified view of polymer, dumbbell, and oligonucleotide DNA nearest-neighbor thermodynamics. Proc Natl Acad Sci USA. 1998;95(4):1460-1465. https://doi.org/10.1073/pnas.95.4 1460.

48. Almada P, Pereira PM, Culley S, et al. Automating multimodal microscopy with NanoJ-Fluidics. Nat Commun. 2019;10(1):1223. https:// doi.org/10.1038/s41467-019-09231-9.

49. Ries J, Kaplan C, Platonova E, Eghlidi H, Ewers H. A simple, versatile method for GFP-based super-resolution microscopy via nanobodies. Nat Methods. 2012;9(6):582-584. https://doi.org/10.1038/nmeth. 1991.

50. Burgert A, Letschert S, Doose S, Sauer M. Artifacts in single-molecule localization microscopy. Histochem Cell Biol. 2015;144(2):123-131. https://doi.org/10.1007/s00418-015-1340-4.

51. Pereira PM, Almada P, Henriques R. High-content 3D multicolor super-resolution localization microscopy. Methods Cell Biol. 2015;125: 95-117. https://doi.org/10.1016/bs.mcb.2014.10.004

52. Chandradoss SD, Haagsma AC, Lee YK, Hwang J-H, Nam J-M, Joo C. Surface passivation for single-molecule protein studies. J Vis Exp. 2014;(86):e50549. https://doi.org/10.3791/50549. 
53. Schindelin J, Arganda-Carreras I, Frise E, et al. Fiji: an open-source platform for biological-image analysis. Nat Methods. 2012;9(7):676682. https://doi.org/10.1038/nmeth.2019.

54. Ester M, Kriegel H-P, Sander J, Xu X. A density-based algorithm for discovering clusters a density-based algorithm for discovering clusters in large spatial databases with noise. KDD'96 Proc Second Int Conf Knowl Discov Data Min. 1996;96(34):226-231. https://doi.org/10. 5555/3001460.3001507.

55. Donoho DL, Johnstone IM. Adapting to unknown smoothness via wavelet shrinkage. J Am Stat Assoc. 1995;90(432):1200. https://doi. org/10.2307/2291512.

56. Broughton SA, Bryan K. Discrete Fourier Analysis and Wavelets. Hoboken, NJ: John Wiley \& Sons, Inc.; 2008. doi:https://doi.org/10.1002/ 9781118032442

57. Ruch DK, Van Fleet PJ. Wavelet Theory. Hoboken, NJ: John Wiley \& Sons, Inc.; 2009. doi:https://doi.org/10.1002/ 9781118165652
58. Stein CM. Estimation of the mean of a multivariate Normal distribution. Ann Stat. 1981;9(6):1135-1151.

\section{SUPPORTING INFORMATION}

Additional supporting information may be found online in the Supporting Information section at the end of this article.

How to cite this article: Pereira PM, Gustafsson N, Marsh M, Mhlanga MM, Henriques R. Super-beacons: Open-source probes with spontaneous tuneable blinking compatible with live-cell super-resolution microscopy. Traffic. 2020;1-11.

https://doi.org/10.1111/tra.12728 